\title{
TVA-based modeling of short-term memory capacity, speed of processing and perceptual threshold in chronic stroke patients undergoing cognitive training: Case-control differences, reliability, and associations with cognitive performance
}

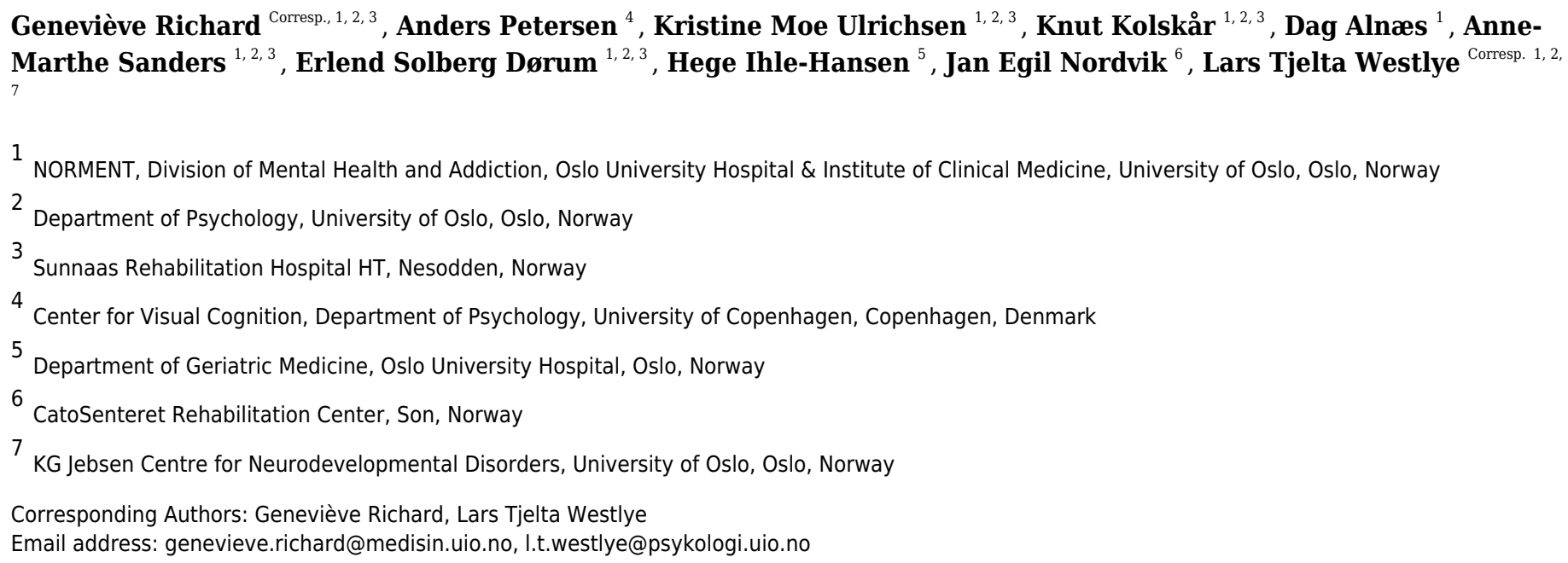

Attentional deficits following stroke are common and pervasive, and are important predictors for functional recovery. Attentional functions comprise a set of specific cognitive processes allowing to attend, filter and select among a continuous stream of stimuli. These mechanisms are fundamental for more complex cognitive functions such as learning, planning and cognitive control, all crucial for daily functioning. The distributed functional neuroanatomy of these processes is a likely explanation for the high prevalence of attentional impairments following stroke, and underscores the importance of a clinical implementation of computational approaches allowing for sensitive and specific modeling of attentional sub-processes. The Theory of Visual Attention (TVA) offers a theoretical, computational, neuronal and practical framework to assess the efficiency of visual selection performance and parallel processing of multiple objects. Here, in order to assess the sensitivity and reliability of TVA parameters reflecting short-term memory capacity $(K)$, processing speed $(C)$ and perceptual threshold $\left(t_{0}\right)$, we used a whole-report paradigm in a cross-sectional case-control comparison and across six repeated assessments over the course of a three-week computerized cognitive training (CCT) intervention in chronic stroke patients ( $>6$ months since hospital admission, NIHSS $\leq 7$ at hospital discharge). Cross-sectional group comparisons documented lower short-term memory capacity, lower 
processing speed and higher perceptual threshold in patients $(n=70)$ compared to agematched healthy controls $(n=140)$. Further, longitudinal analyses in stroke patients during the course of CCT $(n=54)$ revealed high reliability of the TVA parameters, and higher processing speed at baseline was associated with larger cognitive improvement after the intervention. The results support the feasibility, reliability and sensitivity of TVA-based assessment of attentional functions in chronic stroke patients. 
1 TVA-based modeling of short-term memory capacity, speed of processing and perceptual

2 threshold in chronic stroke patients undergoing cognitive training: Case-control

3 differences, reliability, and associations with cognitive performance

4

5 Geneviève Richard ${ }^{\mathrm{a}, \mathrm{b}, \mathrm{c},{ }^{*}}$, Anders Petersen $^{\mathrm{d}}$, Kristine M. Ulrichsen ${ }^{\mathrm{a}, \mathrm{b}, \mathrm{c}}$, Knut Kolskåra, ${ }^{\mathrm{a}, \mathrm{c}, \mathrm{c}}$, Dag

6 Alnæs $^{\mathrm{a}}$, Anne-Marthe Sanders ${ }^{\mathrm{a}, \mathrm{b}, \mathrm{c}}$, Erlend S. Døruma ${ }^{\mathrm{a}, \mathrm{b}, \mathrm{c}}$, Hege Ihle-Hansen ${ }^{\mathrm{e}}$, Jan Egil Nordvik ${ }^{\mathrm{f}}$,

7 Lars T. Westlye $\mathrm{a}^{\mathrm{a}, \mathrm{b}, \mathrm{g} *}$

8

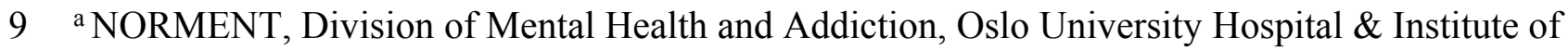

10 Clinical Medicine, University of Oslo, Oslo, Norway

11 b Department of Psychology, University of Oslo, Oslo, Norway

12 c Sunnaas Rehabilitation Hospital HT, Nesodden, Norway

13 d Center for Visual Cognition, Department of Psychology, University of Copenhagen,

14 Copenhagen, Denmark

15 e Department of Geriatric Medicine, Oslo University Hospital, Oslo, Norway

$16{ }^{\mathrm{f}}$ CatoSenteret Rehabilitation Center, Son, Norway

$17 \mathrm{~g}$ KG Jebsen Centre for Neurodevelopmental Disorders, University of Oslo, Oslo, Norway

* Corresponding authors: Geneviève Richard (genevieve.richard@medisin.uio.no) \& Lars T.

Westlye (1.t.westlye@psykologi.uio.no), Department of Psychology, University of Oslo, Po. Box

211094 Blindern, 0317 OSLO, Norway. 


\section{Abstract}

26 Attentional deficits following stroke are common and pervasive, and are important predictors for

27 functional recovery. Attentional functions comprise a set of specific cognitive processes allowing

28 to attend, filter and select among a continuous stream of stimuli. These mechanisms are

29 fundamental for more complex cognitive functions such as learning, planning and cognitive

30 control, all crucial for daily functioning. The distributed functional neuroanatomy of these

31 processes is a likely explanation for the high prevalence of attentional impairments following

32 stroke, and underscores the importance of a clinical implementation of computational approaches

33 allowing for sensitive and specific modeling of attentional sub-processes. The Theory of Visual

34 Attention (TVA) offers a theoretical, computational, neuronal and practical framework to assess

35 the efficiency of visual selection performance and parallel processing of multiple objects. Here,

36 in order to assess the sensitivity and reliability of TVA parameters reflecting short-term memory

37 capacity $(K)$, processing speed $(C)$ and perceptual threshold $\left(t_{0}\right)$, we used a whole-report

38 paradigm in a cross-sectional case-control comparison and across six repeated assessments over

39 the course of a three-week computerized cognitive training (CCT) intervention in chronic stroke

40 patients ( $>6$ months since hospital admission, NIHSS $\leq 7$ at hospital discharge). Cross-sectional

41 group comparisons documented lower short-term memory capacity, lower processing speed and

42 higher perceptual threshold in patients $(n=70)$ compared to age-matched healthy controls

$43(\mathrm{n}=140)$. Further, longitudinal analyses in stroke patients during the course of CCT $(\mathrm{n}=54)$

44 revealed high reliability of the TVA parameters, and higher processing speed at baseline was

45 associated with larger cognitive improvement after the intervention. The results support the 
46 feasibility, reliability and sensitivity of TVA-based assessment of attentional functions in chronic

47 stroke patients.

48 Introduction

49 Attentional deficits following stroke are common, pervasive and persistent (Barker-Collo et al.

50 2010a), likely due to the distributed functional neuroanatomy supporting the range of attentional

51 sub-functions (Rosenberg et al. 2017). Specific functions of attention, such as the ability to

52 rapidly detect changes in perceptual scenes, or internally sustain focus over several stimuli over

53 an extended period, are fundamental to more complex operations supporting everyday functions

54 such as learning, social interactions and cognitive performance in general, and are important

55 predictors for functional recovery in stroke patients (Peers et al. 2020). For instance, attentional

56 functions assessed at hospital discharge has been shown to be relevant for predicting future

57 recovery (Hyndman et al. 2008), sustained visual and auditory attention measured two months

58 after stroke was a strong predictor of long-term motor recovery (Robertson et al. 1997), poorer

59 attentional performance was associated with a more negative impact of stroke on daily

60 functioning (McDowd et al. 2003), and attentional abilities have been associated with language

61 recovery after stroke (Geranmayeh et al. 2014).

62 Whereas the prevalence of attentional deficits following stroke is high, the reported

63 estimates vary depending on assessment tool (Barker-Collo et al. 2010b; Xu et al. 2013), and

64 subtle deficits are most likely underestimated when based on traditional bedside examination

65 (Rinne et al. 2013). Given the high prevalence of attentional impairments in the acute and

66 chronic stages of stroke and the relevance of attentional functions as a predictor of recovery and

67 everyday functions, there is a need to identify specific and reliable behavioral markers of

68 attentional abilities in individual patients. From a cognitive perspective, visual attention broadly 
69 refers to the joint set of cognitive processes that enables efficient and continuous selection and

70 discrimination between competing stimuli from visual scenes of various degrees of complexity,

71 including attentional capacity which comprises speed and storage aspects, and is a core

72 determinant of cognitive performance in general. The cognitive and computational sub-processes

73 involved can be operationalized and assessed using various paradigms. Representing one of the

74 most comprehensive and coherent accounts of attentional capacity, the Theory of Visual

75 Attention (TVA; Bundesen 1990) proposes a number of computational parameters that have been

76 shown to be sensitive to cognitive aging (Espeseth et al. 2014; Habekost et al. 2013; Wiegand et

77 al. 2018), as well as attentional impairments in several brain disorders, including stroke

78 (Habekost 2015; Habekost \& Starrfelt 2009). Briefly, TVA offers a theoretical, computational

79 and practical framework to assess an individual's efficiency of visual selection performance and

80 parallel visual processing of multiple objects. In TVA-based assessments, participants have to

81 either report as many letters as possible (whole-report) or only a subset (partial-report) from a set

82 of briefly displayed letters. TVA assumes that the correctly reported letters are the winners of a

83 race among all the letters in the visual field (later referred to as a biased competition model;

84 Desimone \& Duncan 1995). Moreover, it assumes that the course of the visual encoding process

85 depends on five distinct mathematical parameters: perception threshold $\left(t_{0}\right)$, visual processing

86 speed $(C)$, visual short-term memory capacity $(K)$; visual distractibility $(\alpha)$ and relative

87 attentional weight of each visual object (w) (Habekost 2015). While correlations have been

88 reported between short-term memory capacity $(K)$ and visual processing speed $(C)$ (Habekost et

89 al. 2014), previous studies have demonstrated empirical independence and generally high

90 reliability of the five parameters derived from TVA, with short-term memory capacity $(K)$ 
91 consistently showing highest reliability (Finke et al. 2005; Habekost \& Bundesen 2003;

92 Habekost et al. 2014; Habekost \& Rostrup 2006).

In addition to its attractive psychometric properties, TVA-based parameters are sensitive

94 to attentional dysfunction in clinical groups. Processing speed and short-term memory capacity

95 as measured with TVA have been shown to be selectively impaired in patients with parietal

96 lesions and preserved in patients with frontal lesions (Bublak et al. 2005; Peers et al. 2005).

97 Further, a TVA-based paradigm was shown to be sensitive to asymmetric visual perception after

98 right sided lesions in a group of patients showing minor to no clinical deficits (Habekost \&

99 Rostrup 2006). Moreover, while right hemisphere lesions did not cause deficits in short-term

100 memory capacity, intact white matter connectivity was important for preserved visual short-term

101 memory capacity and ipsilesional processing speed (Habekost \& Rostrup 2007). Together these

102 previous studies highlight the sensitivity of TVA parameters to detect dissociable and subclinical

103 attentional deficits.

104 Here, we combined a cross-sectional case-control comparison in 70 chronic stroke

105 patients who suffered mild to moderate stroke ( $>6$ months since hospital admission, National

106 Institute of Health Stroke Scale (NIHSS; Lyden et al. 2009) $\leq 7$ at hospital discharge) and

107 healthy controls $(n=140)$ with a longitudinal assessment during the course of a computerized

108 cognitive training (CCT), in which 54 of the stroke patients completed an intensive CCT

109 program and were either assigned to an active transcranial direct current stimulation (tDCS) or

110 sham-tDCS condition in order to test for putative beneficial effects of brain stimulation in

111 combination with CCT (Kolskår et al. 2019). We assessed the sensitivity and reliability of TVA

112 parameters reflecting short-term memory capacity $(K)$, processing speed $(C)$ and perceptual

113 threshold $\left(t_{0}\right)$ based on a whole-report behavioral paradigm. In addition, we tested for 
114 associations between TVA parameters of visual attention and various clinical measures,

115 including severity, stroke subtype, and lesion extent and location.

116 Based on the notion that TVA provides sensitive and specific measures of visual attention

117 and that subtle attentional deficits are common and pervasive following even relatively mild

118 strokes, we hypothesized that (1) chronic stroke patients at the group level would show evidence

119 of lower short-term memory capacity, lower processing speed, and higher perceptual threshold

120 compared to healthy peers. Further, assuming that the TVA paradigm provides sensitive

121 measures of subtle attentional deficits (Bublak et al. 2005; Habekost \& Rostrup 2006; Habekost

122 \& Rostrup 2007), we anticipated (2) associations between TVA parameters of visual attention

123 and various clinical measures. More specifically, we hypothesized that higher NIHSS scores

124 would be associated with poorer TVA performance, and tested for associations between TVA

125 parameters and TOAST classification and lesion location. Given that the working memory

126 parameter $K$ consistently has been demonstrated to show high reliability and sensitivity to

127 clinical conditions (Finke et al. 2005; Habekost \& Bundesen 2003; Habekost et al. 2014;

128 Habekost \& Rostrup 2006), we anticipated stronger association with clinical measures for that

129 parameter. Next, we hypothesized that (3) TVA parameters would constitute reliable and

130 sensitive measures of specific attentional functions in chronic stroke patients in a longitudinal

131 context, with the highest reliability found for parameter $K$. Further, based on the concept of

132 cognitive reserve (Shin et al. 2020) and assuming that specific attentional abilities are beneficial

133 for learning and cognitive plasticity, we hypothesized that (4) higher attentional abilities as

134 measured using TVA at baseline would be associated with larger cognitive improvement during

135 the course of the CCT, both for patients receiving active and sham tDCS as part of the

136 intervention protocol. Lastly, we hypothesized that (5) improvement in TVA performance would 
137 be associated with improvement in CCT performance. Based on previous reports showing

138 strongest practice effect for parameter $C$ (Habekost et al. 2014), we expected strongest

139 correlation between improvement in CCT performance score and processing speed.

140 To test these hypotheses, we obtained data using a whole-report behavioral paradigm

141 from chronic stroke patients who were invited to take part of a randomized, double blind study

142 aimed to test the utility of tDCS in combination with CCT to improve cognitive performance

143 following stroke (Kolskår et al. 2019; Richard et al. 2020; Ulrichsen et al. 2019). We used cross-

144 sectional data from 70 chronic stroke patients collected during the first assessment and 140

145 matched controls, as well as longitudinal data (6 sessions) from 54 chronic stroke patients who

146 completed the full study protocol. As tDCS manipulation (sham vs active group) played a central

147 part in the study design, tDCS condition were taken into account in all models.

148

\section{Materials and methods}

150 Table 1 summarizes key demographics and clinical information for the healthy controls and the

151 patient group, including the Mini-Mental Status Examination (MMSE; Strobel \& Engedal 2008), the

152 Montreal Cognitive Assessment (MoCA; Nasreddine et al. 2005), the General Anxiety Disorder-7 (GAD-

153 7; Spitzer et al. 2006) and the Patient Health Questionnaire-9 (PHQ-9; Kroenke et al. 2001). Figure 1

154 depicts a schematic timeline of the study protocol.

155

156

- Insert Table 1 and Figure 1 -

157

Healthy controls

159 Healthy individuals were recruited through advertisement in newspapers, social media and word-

160 of-mouth (Dørum et al. 2019; Richard et al. 2018). Exclusion criteria included history of stroke, 
161 dementia, or other neurologic and psychiatric diseases, alcohol- and substance abuse,

162 medications significantly affecting the nervous system and contraindications for MRI. From a

163 pool of 301 healthy controls who completed the behavioral paradigm and were within the

164 relevant age range (24-81 years), we selected 140 individuals (age 31-81, mean $=67.4, \mathrm{SD}=9.1$,

16555 females) matched by age and sex using the function matchit with the default method nearest

166 from the R package MatchIt (Ho et al. 2011). Here, we employed a ratio of 2:1, in which 2

167 healthy controls were selected for each patient.

168

169 Patient sample

170 Patients admitted to the Stroke Unit at Oslo University Hospital and at Diakonhjemmet Hospital,

171 Oslo, Norway during 2013-2016 were invited to participate in a study with the main aim to test

172 the clinical feasibility of combining CCT and tDCS to improve cognitive function in chronic

173 stroke patients (Kolskår et al. 2019; Richard et al. 2020; Ulrichsen et al. 2019). Stroke was

174 defined as any form of strokes of ischemic or hemorrhagic etiology. We included patients in the

175 chronic stage defined as a minimum of 6 months since hospital admission. Exclusion criteria

176 included transient ischemic attacks (TIA), MRI contraindications and other neurological diseases

177 diagnosed prior to the stroke. Clinical severity was indexed by NIHSS scores at the hospital

178 discharge, stroke subtype was classified using the Trial of Org 10172 in Acute Stroke Treatment

179 (TOAST; Adams et al. 1993) classification system, and a coarse four-class classification of

180 lesion location (left hemisphere, right hemisphere, bilateral and brain stem/cerebellum lesions).

181 NIHSS have been shown to have a good predictive values for the prognosis of patients with

182 acute cerebral infarction (Zhao et al. 2018); however, it has demonstrated limited ability to

183 identify cognitive deficits in acute stroke (Abzhandadze et al. 2020). TOAST has been shown 
184 sensitive to lesion characteristics (Kang et al. 2003); however, there is a lack of studies assessing

185 TOAST sensitivity to cognitive functions and attentional deficits. None of the patients included

186 in this study reported severe visual or linguistic deficits.

187 Seventy-two patients completed TVA-based test at the first assessment and 54 of these

188 patients completed the full protocol; including three MRI brain scan sessions, three main

189 cognitive assessment sessions, which included a broad selection of cognitive tests in addition to

190 the TVA paradigm, one EEG assessment session, and 17 CCT sessions. Beyond the three main

191 cognitive assessment sessions, TVA assessment was repeated weekly during the course of the

192 CCT period. Here, we excluded one patient based on incomplete baseline cognitive assessment

193 (first main assessment) and one due to lack of confirmed stroke resulting in the inclusion of 70

194 patients for the case-control comparisons (age $=24-81$, mean $=67.7, \mathrm{SD}=10.1,20$ females). Of

195 these, all patients who completed the six TVA sessions were included in the longitudinal analysis

$196(\mathrm{~N}=54$, age $=47-82$, mean $=69.7, \mathrm{SD}=7.5,14$ females $)$.

197 The study was approved by the Regional Committee for Medical and Health Research

198 Ethics South-East Norway (2014/694) and conducted in accordance with the Helsinki

199 declaration. All participants signed an informed consent prior to enrollment and received a 200 compensation of $500 \mathrm{NOK}$ for their participation.

201

202

CCT protocol

203 The computerized working memory training program (Cogmed Systems AB, Stockholm,

204 Sweden) consisted of 25 online training sessions. In this study, to increase feasibility, we utilized

20517 sessions over a period of three to four weeks, corresponding to approximately five weekly

206 training sessions (Kolskår et al. 2019; Richard et al. 2020). On average, patients completed two 
207 training sessions combined with tDCS (stimulation was applied during the first 20 minutes of the

208 training session) per week with a minimum of one day between each tDCS session. The

209 remaining CCT sessions were performed at home. Each training session comprised eight

210 different exercises and lasted for about 45 minutes. In total, 10 different tasks targeting verbal

211 and visuospatial working memory were used. The difficulty level of each task was automatically

212 adapted to the participant's performance throughout the intervention.

213

214 tDCS protocol

215 The details of the protocol have been described previously (Kolskår et al. 2019). Briefly,

216 participants were randomly assigned to an active or a sham condition. We used a battery-driven

217 direct current stimulator (Neuroconn DC-STIMULATOR PLUS, neuroConn GmbH, Illmenau,

218 Germany), with the following parameters: DC current $=1 \mathrm{~mA}$, total duration $=20$ minutes,

219 ramp-up $=120$ seconds, fade-out $=30$ seconds, and current density $=28.57 \mu \mathrm{A} / \mathrm{cm} 2$, rubber pads

220 size $=5 \times 7 \mathrm{~cm}$. We used the factory settings for the sham condition, including a ramp-up and a

221 fade-out period. Based on the 10-20 system for the electrode location, the anodal electrode was

222 placed over F3 and the cathodal electrode over O2. The pads were covered with high-conductive

223 gel (Abralyt HiCl, Falk Minow Services Herrsching, Germany) to keep the impedance threshold

224 under $<20 \mathrm{k} \Omega$ and fixated with rubber bands. Side-effects were monitored following each

225 session through self-report forms.

226

227

TVA-based assessment

228 TVA-based modeling was based on data from a whole-report paradigm (Dyrholm et al. 2011;

229 Sperling 1960), in which six red letters from a set of 20 different letters 
230 (ABDEFGHJKLMNOPRSTVXZ) were briefly presented on a circle for either 20, 40, 60, 110,

231 and $200 \mathrm{~ms}$ terminated by a pattern mask or presented for 40 or $200 \mathrm{~ms}$ unmasked. The paradigm

232 was presented on a 24" BenQ XL2430T gaming monitor at a refresh rate set of $100 \mathrm{~Hz}$. The

233 participants were seated at a distance of $60 \mathrm{~cm}$ in a semi-dark room. Participants were instructed

234 to report all the letters they were "fairly certain" of having seen (i.e., to use all available

235 information but refrain from pure guessing). The paradigm comprised 20 practice trials and 140

236 test trials (i.e., 20 trials for each of the seven exposure duration conditions), with an overall

237 duration of approximately 20-25 minutes, including the instruction and the practice trials. TVA

238 parameters $K, C, t_{0}$ were estimated by a maximum-likelihood procedure using the LibTVA

239 toolbox (Dyrholm et al. 2011). The model had 8 degrees of freedom (df): $K, 5 \mathrm{df}$ (the value

240 reported is the expected $K$ given a particular distribution of the probability that on a given trial, $K$

$241=1,2, \ldots, 6) ; C, 1 \mathrm{df} ; t_{0}, 1 \mathrm{df}$; and $\mu$ (additional effective exposure duration for unmasked letters),

$2421 \mathrm{df}$. For those participants whose $t_{0}$ was estimated to be below 0 , we refitted the data fixing $t_{0}$ at

243 0. In addition, the error rate (i.e., the percentage of incorrect letters out of the reported letters)

244 was calculated.

245

246 Processing of Cogmed data

247 We used the same performance improvement scores as in previous publications (Kolskår et al.

248 2019; Richard et al. 2020). Briefly, we used linear modeling with performance as dependent

249 variable and session as independent variable to quantify the changes in performance across the

250 training period for each participant and for each trained task. Next, we performed a principal

251 component analysis (PCA) on the performance improvement scores (zero-centered and 
252 standardized beta estimates from the linear models) and used the first factor as the individual's

253 performance improvement across the trained tasks.

254

255 Statistical analysis

256 Statistical analyses were performed using R version 3.3.3 (2017-03-06) (R Core Team 2017). To

257 test our hypothesis of impaired attentional functions in stroke patients compared to healthy peers, 258 we compared the TVA parameters between groups using linear models with each of the TVA

259 parameters as dependent variables, group (patients and controls) as independent variable, and age

260 and sex as covariates. Since we observed a difference between groups in term of level of

261 education, we performed an additional analysis including education in the model. To control for

262 the number of tests, we employed Bonferroni correction with $\alpha=0.05 / 4$. Cohen's $D$ was

263 calculated using two times the t-value divided by the square root of the degrees of freedom.

264 To test for associations between clinical characteristics and severity at hospital discharge

265 and TVA parameters, we used linear models with each of the TVA parameters as dependent

266 variables and each of the clinical scores (NIHSS, TOAST classification, lesion location) as

267 independent variables, including age and sex as covariates in all models.

268 To assess the reliability of the TVA parameters in a longitudinal context, we estimated

269 the intra-class coefficient (ICC) using ICCest function from the ICC R package (Wolak et al.

2702012 ) across the six sessions.

271 To test if higher attentional performance at baseline (first main assessment) was

272 associated with larger cognitive improvement during the course of the intervention, we

273 conducted four linear models with Cogmed performance gain as dependent variable and each of

274 the TVA parameters as independent variable, including age and sex as covariates in all models. 
275 As a supplemental analysis, we added tDCS group (sham vs experimental) as an additional

276 variable and tested for interactions between tDCS and each of the TVA parameters on cognitive

277 improvement.

278 To test whether improvement in TVA performance scores was associated with

279 improvement in CCT performance, we did the following: First, we estimated TVA performance

280 change using linear modeling with performance as dependent variable and session as

281 independent variable to quantify the changes in performance across the six TVA assessments for

282 each participant and for each TVA parameters. Then, we calculated the correlations between the

283 beta estimates for the TVA parameters and the CCT performance score.

284 Lastly, we investigated whether tDCS condition was associated with improvement rate on

285 TVA using linear mixed effect models (LME) testing for associations between TVA parameters

286 and session (time) by group (active and sham).

287

288 Results

289 Cross-sectional-Case-control

290 Table 1 shows the statistics for the case-control comparisons and Figure 2 depicts the

291 associations between age and each of the TVA parameters for the stroke patients and the healthy

292 controls. Table 2 shows the summary statistics for the four linear models testing for association

293 between TVA parameters and group (case-control comparisons). Briefly, at the group level,

294 patients performed significantly poorer than healthy controls on short-term memory capacity $(K)$,

295 processing speed $(C)$ and perceptual threshold $\left(t_{0}\right)$. Error rate did not significantly differ between

296 groups. In addition, the analysis revealed significant main effects of age on parameters $K, C$ and

$297 t_{0}$ indicating poorer performance for older participants and a significant main effect of sex on $t_{0}$, 
298 indicating higher perceptual threshold in women, but no significant main effect of sex on $K$ and

299 C. Supplementary Table 1 shows the summary statistics for the four linear models testing for

300 association between TVA parameters and group (case-control comparisons) when adding

301 education as a covariates and shows similar association patterns as described above.

302

303

- Insert Figure 2, Table 2 and Table 3 -

304

305 Table 3 shows the summary statistics for the associations between each of the clinical measures

306 and each of the TVA parameters. Briefly, we found no significant associations between clinical

307 measures (NIHSS at hospital discharge, TOAST classification or stroke location) and parameters

$308 K, C, t_{0}$ and error rate.

309

310 Longitudinal - Reliability and change over time

311 Figure 3 shows individual performance for each of the TVA parameters across the six timepoints

312 for each group (sham vs tDCS) together with the global inter-class coefficients (ICCs) ranging

313 from $.58(95 \% \mathrm{CI}=.47-.69)$ for perceptual threshold to $.80(95 \% \mathrm{CI}=.72-.86)$ for short-term

314 memory capacity.

315

316

- Insert Figure 3 and Table 4 -

318 Table 4 shows summary statistics from the four linear models testing for associations between

319 Cogmed performance gain and TVA performance at baseline. Briefly, the analysis revealed one

320 significant association between Cogmed performance gain and processing speed (C) suggesting 
321 that higher processing speed at baseline was associated with larger cognitive gain during the

322 course of the intervention. Beyond this, we found no significant effect of age or sex on

323 performance gain. The analysis including tDCS group (sham and experimental) as an additional

324 variable revealed no significant main effect of tDCS group, nor tDCS group by TVA

325 performance interaction on Cogmed performance gain.

326

327

- Insert Table 5 -

328

329 Table 5 shows summary statistics from the LME models testing for associations between TVA

330 parameters and group (sham and experimental) by session interaction, including age and sex as

331 covariates and participant as random factor. The models revealed robust main effect of session

332 on each of the TVA parameters, suggesting improvement in performance over time. Error rate

333 increased over time. Beyond this, the analyses revealed a main effect of age on processing speed

334 (C). We found no group by session interactions on any of the TVA parameters.

335 Figure 4 shows the correlations between performance improvement over the course of the

336 CCT (Cogmed factor scores) and the change in TVA parameters across the six sessions (beta

337 estimates). There was a positive correlation between improvement in CCT and $K$, as well as the

338 error rate, and a negative correlation with $t_{0}$, suggesting that participants showing a larger

339 improvement in Cogmed also showed an increase in short-term memory capacity $(K)$, made

340 more mistakes across TVA sessions, and decreased their perceptual threshold $\left(t_{0}\right)$. The estimated

341 change in speed of processing $(C)$ across sessions did not show any significant correlations with

342 Cogmed improvement. 


\section{Discussion}

345 Attentional deficits following stroke are prevalent and pervasive, and are important predictors of

346 functional and cognitive recovery. Here, we leveraged the computational framework provided by

347 the TVA to demonstrate poorer storage capacity, lower processing speed and higher visual

348 threshold in chronic stroke patients compared to age-matched healthy controls. Further, we

349 demonstrated high reliability of the TVA parameters in stroke patients across six test sessions.

350 We also showed that higher processing speed at baseline, as indexed by the $C$ parameter from

351 TVA, was associated with larger cognitive improvement during the course of cognitive training.

352 Based on the notion that TVA provides sensitive and specific measures of visual attention

353 and that attentional deficits are common and pervasive following even relatively mild strokes, we

354 first tested whether chronic stroke patients would show reduced performance compared to age-

355 matched healthy controls in $K, C$ and $t_{0}$. Our results demonstrated that, at group level, patients

356 who suffered mild to moderate stroke, defined here with a NIHSS score below or equal to seven

357 at the hospital discharge, showed reduced performance compared to age-matched controls,

358 suggesting that attentional deficits are present also in patients suffering from relatively mild

359 stroke. These effects could not be explained by a difference in education level between groups,

360 and are in line with previous literature showing that TVA is sensitive to subtle deficits (Bublak et

361 al. 2005; Habekost \& Bundesen 2003; Habekost \& Rostrup 2006; Habekost \& Rostrup 2007).

362 Next, to the extent that clinical characteristics and severity of the stroke at hospital discharge are

363 sensitive to visual attention, we tested whether higher clinical burden was associated with lower

364 performance on TVA as measured by $K, C$ and $t_{0}$. We found no associations between NIHSS at

365 hospital discharge, suggesting that, among patients with relatively mild strokes, the severity of

366 the stroke as measured by NIHSS is not a strong predictor of short-term memory capacity, 
367 processing speed, nor perceptual threshold as measured by TVA in a chronic stage. These

368 findings are in line with a recent study reporting poor predictive value of NIHSS obtained at the

369 hospital admission for cognitive functions in the acute phase (Abzhandadze et al. 2020). Further,

370 we found no significant association between TOAST nor location of the stroke, and performance

371 on TVA. Thus, neither stroke severity as measured by NIHSS at hospital discharge, nor the

372 etiology of stroke based on TOAST or stroke location provided predictive value of attentional

373 performance measured by TVA in a chronic stage, highlighting the complexity of the clinical

374 etiology of long-term attentional impairments following stroke.

375 In line with previous findings using three test sessions in healthy individuals (Habekost et

376 al. 2014) and studies assessing internal reliability (Finke et al. 2005; Habekost \& Rostrup 2006),

377 our results demonstrate highest reliability for the $K$ parameter, and fairly good reliability for $C$

378 and $t_{0}$ across the six TVA sessions in chronic stroke patients. These findings are encouraging as

379 they support both the feasibility and reliability of computational behavioral approaches in a

380 clinical setting, and provide support for previous cross-sectional studies implementing a similar

381 paradigm and modeling approach.

382 To test the extent that attentional abilities facilitate response to cognitive training, we

383 tested whether higher attentional abilities as measured by the TVA at baseline were associated

384 with larger improvement in response to cognitive training during the course of the intervention.

385 Our results revealed a significant association between processing speed at baseline and

386 performance improvement over the course of the intervention, indicating that patients with

387 higher processing speed showed larger cognitive improvements, which is in line with the

388 cognitive reserve hypothesis (Shin et al. 2020). In contrast, visual memory capacity and

389 perceptual threshold at baseline were not significantly associated with cognitive improvement. A 
390 previous study in 68 healthy young individuals using three test sessions with one week interval

391 reported significant improvement for processing speed, visual memory capacity and perceptual

392 threshold, with the strongest practice effects for processing speed (Habekost et al. 2014). These

393 previous findings are largely in line with our observations of substantial practice effect for all

394 three TVA parameters across six test sessions in chronic stroke patients during the course of

395 CCT. Future studies including appropriate control conditions are needed to assess to which

396 degree cognitive training or other interventions influence TVA parameters beyond simple

397 practice effects.

398 Further, we tested whether improvement in TVA performance was associated with 399 improvement in CCT performance by correlating performance improvement over the course of 400 the CCT (Cogmed factor scores) and the change in TVA parameters across the six sessions (beta

401 estimates). The results suggested associations between both short-term memory capacity $(K)$ and

402 processing threshold $\left(t_{0}\right)$ and improvement in CCT performance, suggesting that whereas

403 processing speed $(C)$ was not significantly associated with CCT improvement, participants

404 showing a larger improvement in Cogmed also showed an increase in short-term memory

405 capacity $(K)$, a decreased their perceptual threshold $\left(t_{0}\right)$, and an increased error rate across TVA 406 sessions.

407 Our results jointly demonstrate that processing speed as indexed by the $C$ parameter is

408 both reliable, sensitive to case-control differences between stroke patients and age-matched

409 healthy peers, and associated with response to cognitive training in chronic stroke patients. These

410 findings support a clinical implementation of computational cognitive approaches in general and

411 of TVA specifically in future stroke studies. 
412 In line with one of the aims of the intervention, we also tested for effects of tDCS on

413 cognitive improvement and interactions between attentional abilities as measured by TVA and

414 tDCS on cognitive improvement. In addition, we tested for tDCS by time interaction on TVA

415 performance to assess whether the experimental conditions would result in differential

416 improvement in TVA performance over time. Here, corroborating previous publications using

417 the same sample (Kolskår et al. 2019; Richard et al. 2020), we found no significant associations

418 between tDCS group (sham and experimental) and cognitive improvement, nor tDCS group by

419 session interactions on TVA performance, providing no support for a beneficial effect of tDCS.

420 These results are in lines with a recent review and meta-analysis study reporting limited effect of

421 tDCS in the context of memory training (Galli et al. 2019).

422 Several methodological considerations need to be highlighted while interpreting our

423 results. First, as previously emphasized (Richard et al. 2020), patients included in this study

424 represent a high functioning group with relatively mild cognitive deficits and presumably better

425 prognosis compared to patients with more severe symptoms, limiting the generalizability of our

426 findings. It is possible that the current analysis would have revealed stronger associations

427 between clinical variables and TVA parameters in a sample including a broader range of stroke

428 severities and cognitive impairments, or if using different stroke classification schemes. The

429 labor-intensity of the current intervention and imaging study was demanding for the individual

430 patient, preventing the study from sampling a wider range of the stroke severity spectrum. Future

431 studies are needed to test if the value of TVA and other computational behavioral approaches

432 may be particularly high in relatively well functioning samples where the cognitive deficits are

433 assumed to be subtle (Ulrichsen et al. 2019). Moreover, the cross-sectional design prevents us

434 from determining whether the group differences between patients and controls in TVA 
435 parameters primarily result from the stroke, or were present prior to the stroke. Further, the lack

436 of control group performing an alternative or sham CCT in the longitudinal study does not allow

437 us to separate beneficial effects of Cogmed on TVA performance from learning effects in

438 response to repeated TVA assessments. Indeed, previous studies have reported significant

439 practice effect on all three TVA parameters, with the strongest effect observed for processing

440 speed (Habekost et al. 2014). Further, regarding the reliability estimates, although the reliability

441 coefficients for all TVA parameters were fairly high and in line with previous studies, it is

442 possible that the individual variability in the CCT gain could have led to underestimating ICC for

443 the different TVA parameters. Thus, the reported values should be considered lower-bound

444 estimates. Lastly, while none of the patients reported severe visual or linguistic impairments,

445 future studies should apply relevant clinical assessments to rule out an impact of subtle visual or

446 language deficits on task performance, which will be important to assess the validity of TVA

447 performance as an indicator of attentional impairments.

\section{Conclusions}

450 In conclusion, we have assessed the sensitivity and reliability of TVA parameters assessing 451 short-term memory capacity $(K)$, processing speed $(C)$ and perceptual threshold $\left(t_{0}\right)$ derived

452 using a whole-report behavioral paradigm in a cross-sectional case-control comparison and 453 longitudinal assessment during the course of a CCT scheme in chronic patients who suffered 454 mild stroke. Our results demonstrate poorer $K$, lower $C$ and higher $t_{0}$ in chronic stroke patients 455 compared to healthy controls. Further, we demonstrated high reliability of the TVA parameters 456 in stroke patients across six test sessions, and showed that higher $C$ at baseline was associated 457 with larger cognitive improvement over the course of cognitive training. Thus, a clinically 
458 feasible implementation of TVA-based assessment offers sensitive and reliable computational

459 parameters of short-term memory capacity, speed of processing and visual threshold in chronic

460 stroke patients.

461

462

\section{References}

463

464

465

466

467

468

469

470

471

472

473

474

475

476

477

478

479

480

481

482

483

484

485

486

487

488

489

490

491

492

493

494

495

496

497
Abzhandadze T, Reinholdsson M, and Sunnerhagen KS. 2020. NIHSS is not enough for cognitive screening in acute stroke: A cross-sectional, retrospective study. Sci Rep 10:534. 10.1038/s41598-019-57316-8

Barker-Collo S, Feigin VL, Parag V, Lawes CM, and Senior H. 2010a. Auckland Stroke Outcomes Study. Part 2: Cognition and functional outcomes 5 years poststroke. Neurology 75:1608-1616. 10.1212/WNL.0b013e3181fb44c8

Barker-Collo SL, Feigin VL, Lawes CM, Parag V, and Senior H. 2010b. Attention deficits after incident stroke in the acute period: frequency across types of attention and relationships to patient characteristics and functional outcomes. Top Stroke Rehabil 17:463-476. 10.1310/tsr1706-463

Bublak P, Finke K, Krummenacher J, Preger R, KyllingsbÆK S, MÜLler HJ, and Schneider WX. 2005. Usability of a theory of visual attention (TVA) for parameter-based measurement of attention II: Evidence from two patients with frontal or parietal damage. Journal of the International Neuropsychological Society 11. $10.1017 / \mathrm{s} 1355617705050988$

Bundesen C. 1990. A theory of visual attention. Psychol Rev 97:523-547.

Desimone R, and Duncan J. 1995. Neural mechanisms of selective visual attention. Annu Rev Neurosci 18:193-222. 10.1146/annurev.ne.18.030195.001205

Dørum ES, Kaufmann T, Alnæs D, Richard G, Kolskår KK, Engvig A, Sanders A-M, Ulrichsen K, Ihle-Hansen H, Nordvik JE, and Westlye LT. 2019. Functional brain network modeling in sub-acute stroke patients and healthy controls during rest and continuous attentive tracking. bioRxiv. 10.1101/644765

Dyrholm M, Kyllingsbæk S, Espeseth T, and Bundesen C. 2011. Generalizing parametric models by introducing trial-by-trial parameter variability: The case of TVA. Journal of Mathematical Psychology 55:416-429. 10.1016/j.jmp.2011.08.005

Espeseth T, Vangkilde SA, Petersen A, Dyrholm M, and Westlye LT. 2014. TVA-based assessment of attentional capacities-associations with age and indices of brain white matter microstructure. Front Psychol 5:1177. 10.3389/fpsyg.2014.01177

Finke K, Bublak P, Krummenacher J, Kyllingsbaek S, Muller HJ, and Schneider WX. 2005. Usability of a theory of visual attention (TVA) for parameter-based measurement of attention I: evidence from normal subjects. J Int Neuropsychol Soc 11:832-842. $10.1017 / \mathrm{s} 1355617705050976$

Galli G, Vadillo MA, Sirota M, Feurra M, and Medvedeva A. 2019. A systematic review and meta-analysis of the effects of transcranial direct current stimulation (tDCS) on episodic memory. Brain Stimul 12:231-241. 10.1016/j.brs.2018.11.008

PeerJ reviewing PDF | (2019:08:40638:2:0:NEW 14 Aug 2020) 
498

499

500

501

502

503

504

505

506

507

508

509

510

511

512

513

514

515

516

517

518

519

520

521

522

523

524

525

526

527

528

529

530

531

532

533

534

535

536

537

538

539

540

541

542

Geranmayeh F, Brownsett SL, and Wise RJ. 2014. Task-induced brain activity in aphasic stroke patients: what is driving recovery? Brain 137:2632-2648. 10.1093/brain/awu163

Habekost T. 2015. Clinical TVA-based studies: a general review. Front Psychol 6:290. 10.3389/fpsyg.2015.00290

Habekost T, and Bundesen C. 2003. Patient assessment based on a theory of visual attention (TVA): subtle deficits after a right frontal-subcortical lesion. Neuropsychologia 41:1171-1188. 10.1016/s0028-3932(03)00018-6

Habekost T, Petersen A, and Vangkilde S. 2014. Testing attention: comparing the ANT with TVA-based assessment. Behav Res Methods 46:81-94. 10.3758/s13428-013-0341-2

Habekost T, and Rostrup E. 2006. Persisting asymmetries of vision after right side lesions. Neuropsychologia 44:876-895. 10.1016/j.neuropsychologia.2005.09.002

Habekost T, and Rostrup E. 2007. Visual attention capacity after right hemisphere lesions. Neuropsychologia 45:1474-1488. 10.1016/j.neuropsychologia.2006.11.006

Habekost T, and Starrfelt R. 2009. Visual attention capacity: a review of TVA-based patient studies. Scand J Psychol 50:23-32. 10.1111/j.1467-9450.2008.00681.x

Habekost T, Vogel A, Rostrup E, Bundesen C, Kyllingsbaek S, Garde E, Ryberg C, and Waldemar G. 2013. Visual processing speed in old age. Scand J Psychol 54:89-94. 10.1111/sjop.12008

Ho DE, Imai K, King G, and Stuart EA. 2011. MatchIt: Nonparametric Preprocessing for Parametric Causal Inference. Journal of Statistical Software 42:1-28.

Hyndman D, Pickering RM, and Ashburn A. 2008. The influence of attention deficits on functional recovery post stroke during the first 12 months after discharge from hospital. J Neurol Neurosurg Psychiatry 79:656-663. 10.1136/jnnp.2007.125609

Kang DW, Chalela JA, Ezzeddine MA, and Warach S. 2003. Association of ischemic lesion patterns on early diffusion-weighted imaging with TOAST stroke subtypes. Arch Neurol 60:1730-1734. 10.1001/archneur.60.12.1730

Kolskår KK, Richard G, Alnæs D, Dørum ES, Sanders A-M, Ulrichsen KM, Sánchez JM, IhleHansen H, Nordvik JE, and Westlye LT. 2019. A randomized, double blind study of computerized cognitive training in combination with transcranial direct currentstimulation (tDCS) in stroke patients-utility of fMRI as marker for training outcome. 10.1101/603985

Kroenke K, Spitzer RL, and Williams JB. 2001. The PHQ-9: validity of a brief depression severity measure. J Gen Intern Med 16:606-613. 10.1046/j.15251497.2001.016009606.x

Lyden P, Raman R, Liu L, Emr M, Warren M, and Marler J. 2009. National Institutes of Health Stroke Scale certification is reliable across multiple venues. Stroke 40:25072511. 10.1161/STROKEAHA.108.532069

McDowd JM, Filion DL, Pohl PS, Richards LG, and Stiers W. 2003. Attentional abilities and functional outcomes following stroke. J Gerontol B Psychol Sci Soc Sci 58:P45-53. 10.1093/geronb/58.1.p45

Nasreddine ZS, Phillips NA, Bedirian V, Charbonneau S, Whitehead V, Collin I, Cummings JL, and Chertkow H. 2005. The montreal cognitive assessment, MoCA: A brief screening tool for mild cognitive impairment. Journal of the American Geriatrics Society 53:695-699. DOI 10.1111/j.1532-5415.2005.53221.x 
543

544

545

546

547

548

549

550

551

552

553

554

555

556

557

558

559

560

561

562

563

564

565

566

567

568

569

570

571

572

573

574

575

576

577

578

579

580

581

582

583

584

585

586

587

588

Peers PV, Astle DE, Duncan J, Murphy FC, Hampshire A, Das T, and Manly T. 2020. Dissociable effects of attention vs working memory training on cognitive performance and everyday functioning following fronto-parietal strokes. Neuropsychol Rehabil 30:1092-1114. 10.1080/09602011.2018.1554534

Peers PV, Ludwig CJ, Rorden C, Cusack R, Bonfiglioli C, Bundesen C, Driver J, Antoun N, and Duncan J. 2005. Attentional functions of parietal and frontal cortex. Cereb Cortex 15:1469-1484. 10.1093/cercor/bhi029

R Core Team. 2017. R: A Language and Environment for Statistical Computing. Vienna, Austria: R Foundation for Statistical Computing.

Richard G, Kolskar K, Sanders AM, Kaufmann T, Petersen A, Doan NT, Monereo Sanchez J, Alnaes D, Ulrichsen KM, Dorum ES, Andreassen OA, Nordvik JE, and Westlye LT. 2018. Assessing distinct patterns of cognitive aging using tissue-specific brain age prediction based on diffusion tensor imaging and brain morphometry. peerj 6:e5908. 10.7717/peerj.5908

Richard G, Kolskar K, Ulrichsen KM, Kaufmann T, Alnaes D, Sanders AM, Dorum ES, Monereo Sanchez J, Petersen A, Ihle-Hansen H, Nordvik JE, and Westlye LT. 2020. Brain age prediction in stroke patients: Highly reliable but limited sensitivity to cognitive performance and response to cognitive training. Neuroimage Clin 25:102159. 10.1016/j.nicl.2019.102159

Rinne P, Hassan M, Goniotakis D, Chohan K, Sharma P, Langdon D, Soto D, and Bentley P. 2013. Triple dissociation of attention networks in stroke according to lesion location. Neurology 81:812-820. 10.1212/WNL.0b013e3182a2ca34

Robertson IH, Ridgeway V, Greenfield E, and Parr A. 1997. Motor recovery after stroke depends on intact sustained attention: a 2-year follow-up study. Neuropsychology 11:290-295.

Rosenberg MD, Finn ES, Scheinost D, Constable RT, and Chun MM. 2017. Characterizing Attention with Predictive Network Models. Trends Cogn Sci 21:290-302. 10.1016/j.tics.2017.01.011

Shin M, Sohn MK, Lee J, Kim DY, Lee SG, Shin YI, Oh GJ, Lee YS, Joo MC, Han EY, Han J, Ahn J, Chang WH, Shin MA, Choi JY, Kang SH, Kim Y, and Kim YH. 2020. Effect of Cognitive Reserve on Risk of Cognitive Impairment and Recovery After Stroke: The KOSCO Study. Stroke 51:99-107. 10.1161/STROKEAHA.119.026829

Sperling G. 1960. The information available in brief visual presentations. Psychological Monographs: General and Applied 74:1-29. 10.1037/h0093759

Spitzer RL, Kroenke K, Williams JB, and Lowe B. 2006. A brief measure for assessing generalized anxiety disorder: the GAD-7. Arch Intern Med 166:1092-1097. 10.1001/archinte.166.10.1092

Strobel C, and Engedal K. 2008. Norsk revidert mini mental status evaluering (MMSE-NR). Ulrichsen KM, Alnæs D, Kolskår KK, Richard G, Sanders A-M, Dørum ES, Ihle-Hansen H, Pedersen ML, Tornås S, Nordvik JE, and Westlye LT. 2019. Dissecting the cognitive phenotype of post-stroke fatigue using drift diffusion modeling of sustained attention. 10.1101/582502

Wiegand I, Lauritzen MJ, Osler M, Mortensen EL, Rostrup E, Rask L, Richard N, Horwitz A, Benedek K, Vangkilde S, and Petersen A. 2018. EEG correlates of visual short-term memory in older age vary with adult lifespan cognitive development. Neurobiol Aging 62:210-220. 10.1016/j.neurobiolaging.2017.10.018

Peer) reviewing PDF | (2019:08:40638:2:0:NEW 14 Aug 2020) 
589 Wolak ME, Fairbairn DJ, and Paulse YR. 2012. Guidelines for Estimating Repeatability.

$590 \quad$ Methods in Ecology and Evolution 3:129-137.

591 Xu XD, Ren HY, Prakash R, Vijayadas, and Kumar R. 2013. Outcomes of neuropsychological

592 interventions of stroke. Ann Indian Acad Neurol 16:319-328. 10.4103/0972-

$593 \quad 2327.116909$

594 Zhao XJ, Li QX, Liu TJ, Wang DL, An YC, Zhang J, Peng YB, Chen RY, Chang LS, Wang Y, Zhang

595 L, Fan HY, Wang XJ, and Zheng FX. 2018. Predictive values of CSS and NIHSS in the prognosis of patients with acute cerebral infarction: A comparative analysis.

598 Medicine (Baltimore) 97:e12419. 10.1097/MD.0000000000012419 
Figure 1

Schematic timeline of the study protocol.

Assessment refers to the three main cognitive assessment sessions performed prior to and following the intervention. Waiting period refers to the period between the first and second assessment without any active intervention. TVA: Theory of visual attention. tDCS:

transcranial direct current stimulation.

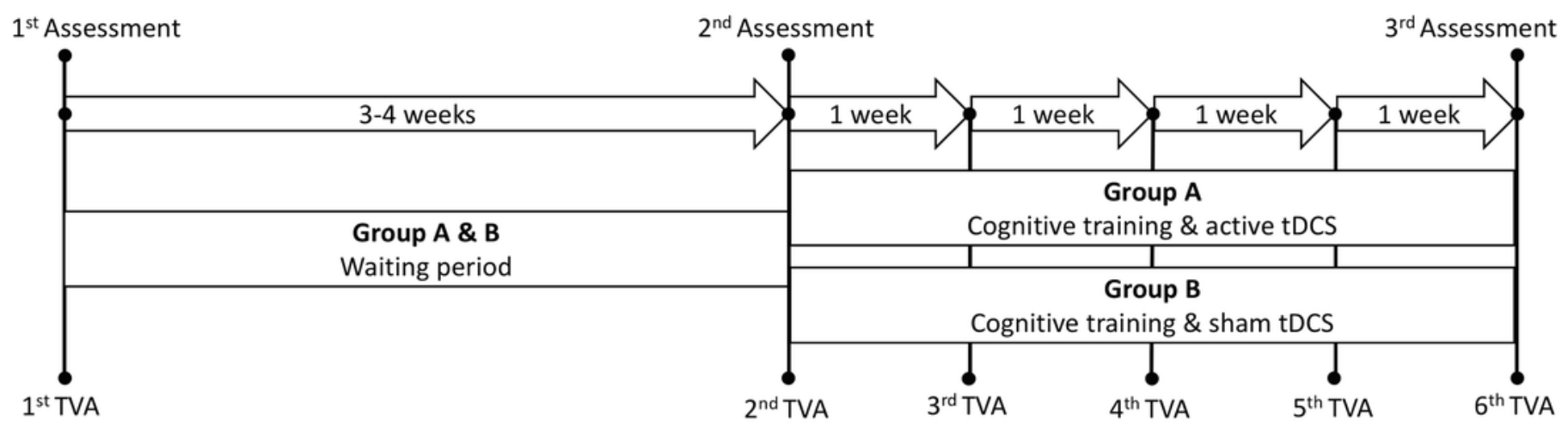


Figure 2

Pearson correlations between age and TVA parameters for each of the two groups independently.

(A) Short-term memory capacity (K). (B) Perceptual processing speed (C). (C) Perceptual threshold $\left(t_{0}\right)$. (D) Error rate. HC: Healthy controls. IVS: Stroke patients.
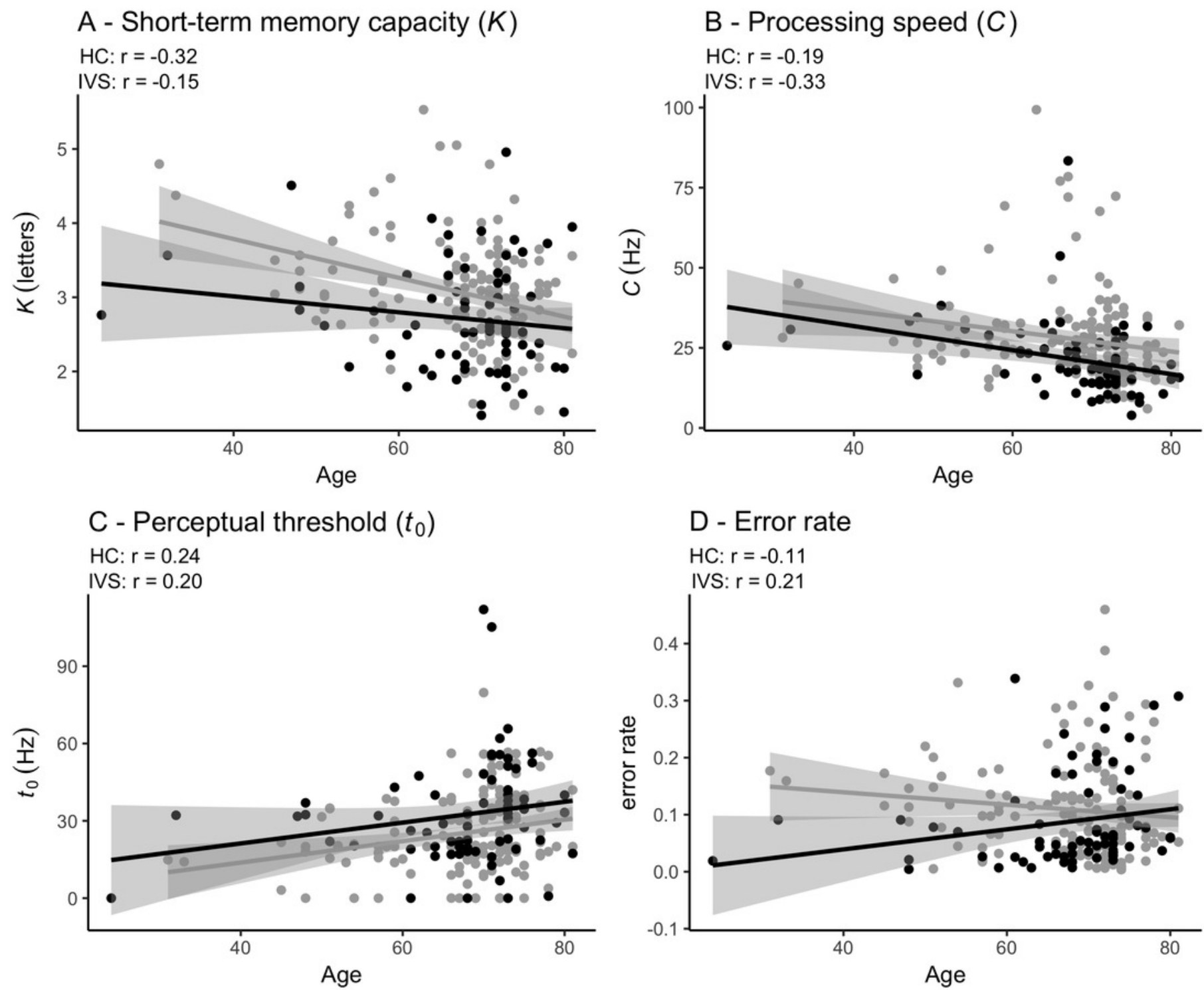

$\mathrm{Dx} \leadsto \mathrm{HC} \leadsto \mathrm{IVS}$ 


\section{Figure 3}

Individual performance for each of the TVA parameters across the six timepoints for each group (sham vs tDCS).

Reliability of each TVA parameter is indicated by the intra-class coefficient (ICC) with $95 \%$ confidence interval $(\mathrm{CI})$. TVA: Theory of visual attention. tDCS: transcranial direct current stimulation. (A) Short-term memory capacity (K). (B) Perceptual processing speed (C). (C) Perceptual threshold $\left(t_{0}\right)$. (D) Error rate. 

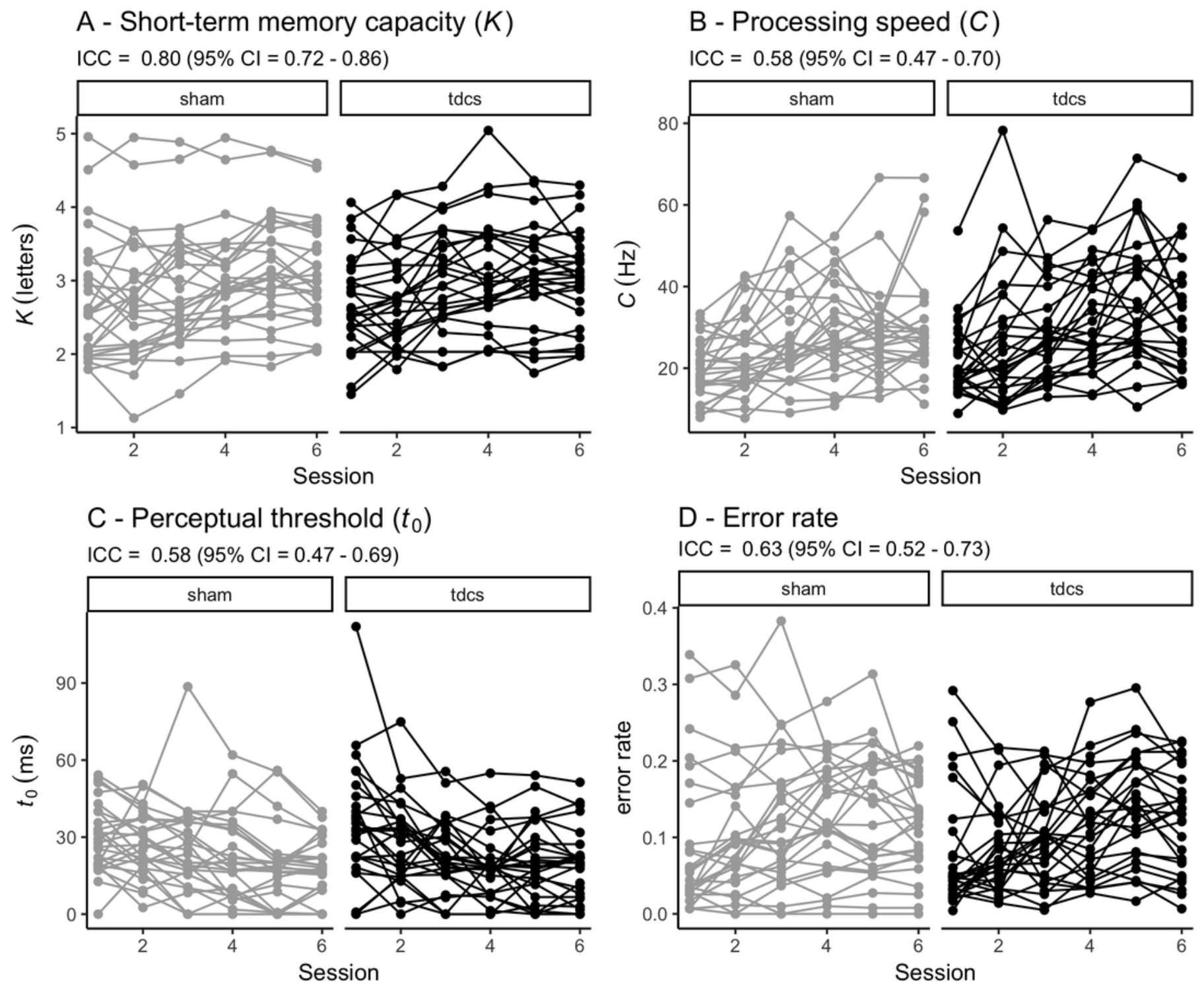

group $\rightarrow-$ sham $\rightarrow$ tdcs 


\section{Figure 4}

Associations between each of the TVA parameter improvement scores (beta estimates) and the Cogmed factor scores for each individuals (on the right).

(A) Correlations between performance improvement over the course of the CCT (Cogmed factor scores) and the change in TVA parameters across the six sessions (beta estimates). The $-\log _{10}(p$-value) matrix of the correlations between performance improvement over the course of the CCT (Cogmed factor scores) and the change in TVA parameters across the six sessions (beta estimates). (C) Associations between change in short-term memory capacity (K) (beta estimates) and the Cogmed factor score. (D) Associations between change in processing speed $(C)$ (beta estimates) and the Cogmed factor scores. (E) Associations between change in perceptual threshold $\left(t_{0}\right)$ (beta estimates) and the Cogmed factor scores. (F) Associations between change in error rate (beta estimates) and the Cogmed factor scores. 

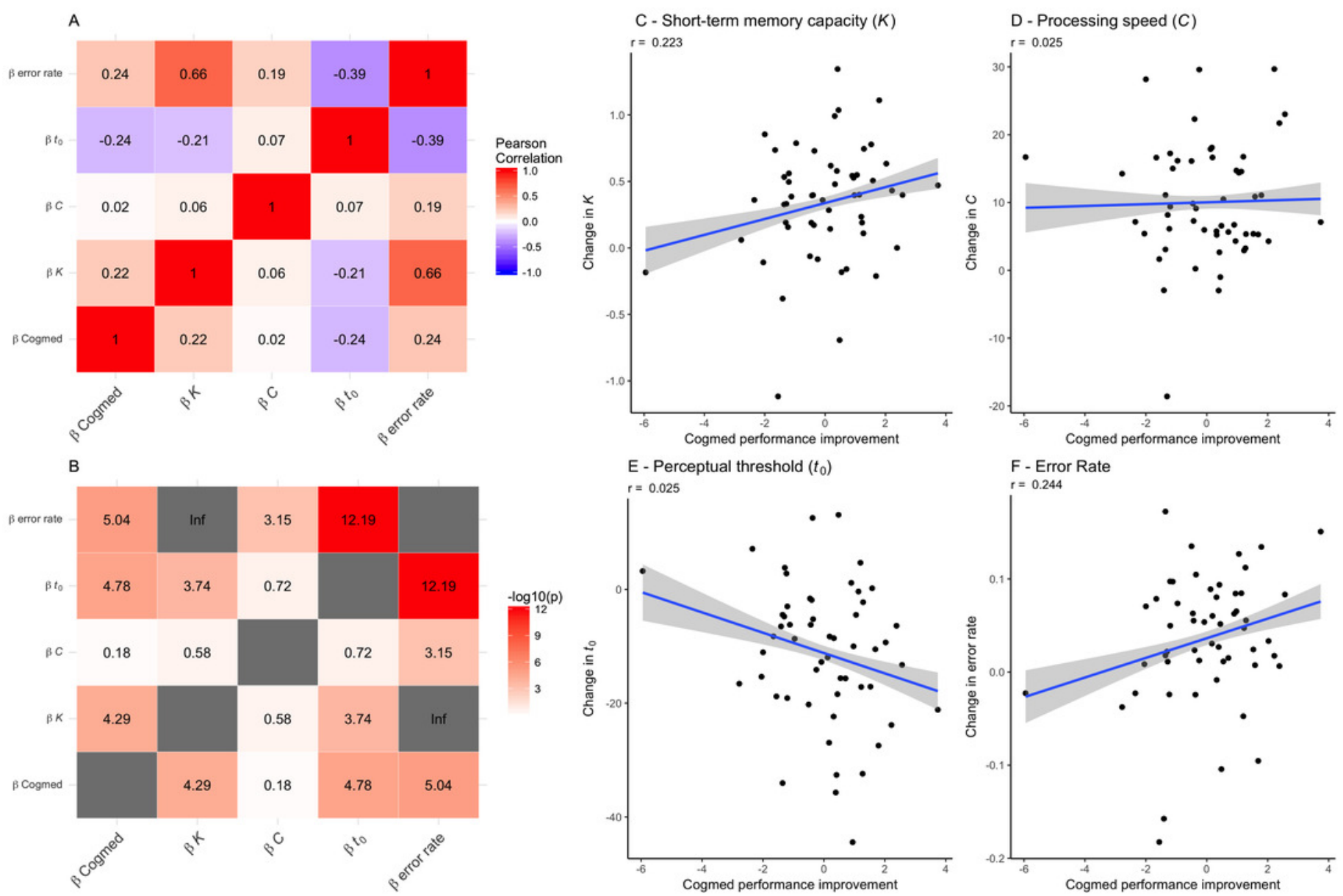


\section{Table $\mathbf{1}$ (on next page)}

Demographics, descriptive statistics and patients sample characteristics.

${ }^{1}$ NIHSS score at hospital discharge. ${ }^{2}$ One patient had intracerebral hemorrhage. (Kolskår et al.

2019; Richard et al. 2020; Ulrichsen et al. 2019) ${ }^{3}$ Chi square statistics. *Significant after Bonferroni correction. SD = standard deviation. $\mathrm{K}=$ short-term memory capacity. $\mathrm{C}=$ perceptual processing speed. $\mathrm{t}_{0}=$ perceptual threshold. MMSE $=$ Mini-Mental Status Examination. MoCA = Montreal Cognitive Assessment. GAD-7 = General Anxiety Disorder-7. PHQ-9 = Patient Health Questionnaire-9. Im = linear model. 


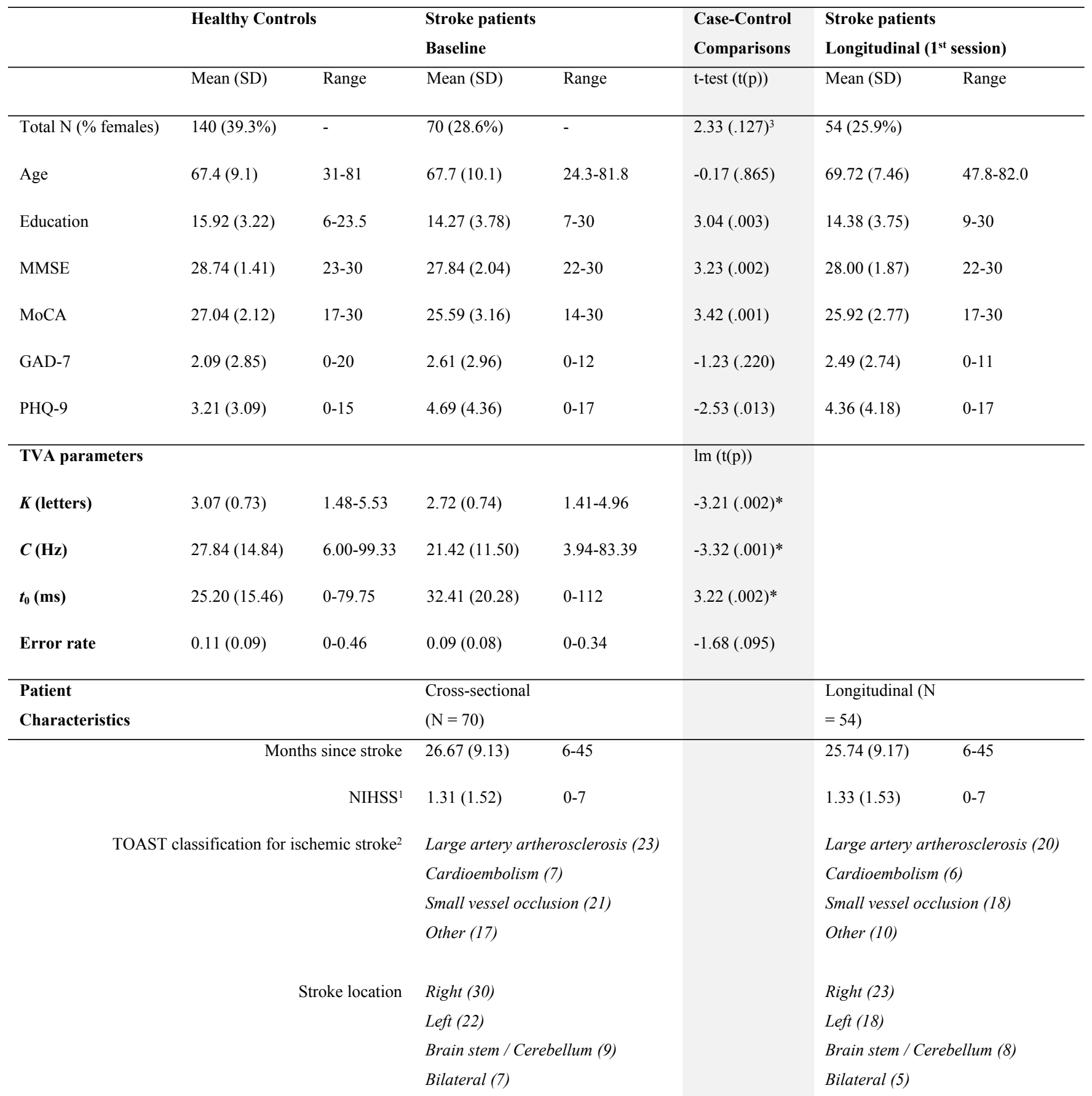

1 Table 1. Demographics, descriptive statistics and patients sample characteristics. ${ }^{1}$ NIHSS score at 2 hospital discharge. ${ }^{2}$ One patient had intracerebral hemorrhage. (Kolskår et al. 2019; Richard et al. 2020; 3 Ulrichsen et al. 2019) ${ }^{3}$ Chi square statistics. *Significant after Bonferroni correction. SD = standard 4 deviation. $K=$ short-term memory capacity. $C=$ perceptual processing speed. $t_{0}=$ perceptual threshold. $5 \mathrm{MMSE}=$ Mini-Mental Status Examination. MoCA = Montreal Cognitive Assessment. GAD-7 = General 6 Anxiety Disorder-7. PHQ-9 = Patient Health Questionnaire-9. $1 \mathrm{~m}=$ linear model. 
9 Kolskår KK, Richard G, Alnæs D, Dørum ES, Sanders A-M, Ulrichsen KM, Sánchez JM, IhleHansen H, Nordvik JE, and Westlye LT. 2019. A randomized, double blind study of computerized cognitive training in combination with transcranial direct currentstimulation (tDCS) in stroke patients-utility of fMRI as marker for training outcome. 10.1101/603985

Richard G, Kolskar K, Ulrichsen KM, Kaufmann T, Alnaes D, Sanders AM, Dorum ES, Monereo Sanchez J, Petersen A, Ihle-Hansen H, Nordvik JE, and Westlye LT. 2020. Brain age prediction in stroke patients: Highly reliable but limited sensitivity to cognitive performance and response to cognitive training. Neuroimage Clin 25:102159. 10.1016/j.nicl.2019.102159

Ulrichsen KM, Alnæs D, Kolskår KK, Richard G, Sanders A-M, Dørum ES, Ihle-Hansen H, Pedersen ML, Tornås S, Nordvik JE, and Westlye LT. 2019. Dissecting the cognitive phenotype of post-stroke fatigue using drift diffusion modeling of sustained attention. $10.1101 / 582502$ 


\section{Table 2 (on next page)}

Summary statistics for the linear models testing for association between TVA parameters (dependent variables) and group (case-control comparisons.

*Significant after Bonferroni correction. $K=$ short-term memory capacity. $C$ = perceptual processing speed. $t_{0}=$ perceptual threshold. Estimate $=$ unstandardized regression coefficients $b$. std.error $=$ standard error. $t=t$-value. $p=p$-value. Cohen's D was calculated using two times the t-value divided by the square root of the degrees of freedom. 
1

\begin{tabular}{lllll}
\hline Parameter & & group & age & sex \\
\hline$K$ & estimate (std.error) & $-0.334(0.104)$ & $-0.020(0.005)$ & $-0.138(0.102)$ \\
& $t(p)$ & $-3.207(0.002)^{*}$ & $-3.910(<.001)^{*}$ & $-1.349(0.179)$ \\
& Cohen's d & -0.447 & -0.545 & -0.188 \\
$C$ & estimate (std.error) & $-6.585(1.983)$ & $-0.337(0.099)$ & $2.368(1.951)$ \\
& $t(p)$ & $-3.321(0.001)^{*}$ & $-3.389(0.001)^{*}$ & $1.214(0.226)$ \\
\multirow{4}{*}{$t_{0}$} & Cohen's d & -0.463 & -0.472 & 0.169 \\
& estimate (std.error) & $7.828(2.435)$ & $0.407(0.122)$ & $-6.684(2.395)$ \\
& $t(p)$ & $3.215(0.002)^{*}$ & $3.334(0.001)^{*}$ & $-2.790(0.006)^{*}$ \\
error rate & Cohen's d & 0.448 & 0.465 & -0.389 \\
& estimate (std.error) & $-0.021(0.013)$ & $0.000(0.001)$ & $-0.003(0.012)$ \\
& $t(p)$ & $-1.676(0.095)$ & $-0.015(0.988)$ & $-0.273(0.785)$ \\
& Cohen's d & -0.234 & -0.002 & -0.038 \\
\hline
\end{tabular}

2 Table 2. Summary statistics for the linear models testing for association between TVA parameters (dependent variables) and group (case-control comparisons) *Significant after Bonferroni correction.

$4 K=$ short-term memory capacity. $C=$ perceptual processing speed. $t_{0}=$ perceptual threshold. Estimate $=$

5 unstandardized regression coefficients $b$. std.error $=$ standard error. $t=t$-value. $p=p$-value. Cohen's D

6 was calculated using two times the $t$-value divided by the square root of the degrees of freedom. 


\section{Table 3 (on next page)}

Summary statistics for the associations between each of the TVA parameters (dependent variables) and each of the clinical measures.

*Significant after Bonferroni correction. ${ }^{1}$ Main effect of TVA parameters on Cogmed performance. $K=$ short-term memory capacity. $C=$ perceptual processing speed. $t_{0}=$

perceptual threshold. NIHSS $=$ National Institute of Health Stroke Scale. TOAST $=$ Trial of Org 10172 in Acute Stroke Treatment (TOAST) classification system. location = coarse four-class classification of lesion location (left hemisphere, right hemisphere, bilateral and brain stem/cerebellum lesions). Estimate $=$ unstandardized regression coefficients $b$. std.error $=$ standard error. $\mathrm{t}=\mathrm{t}$-value. $\mathrm{p}=\mathrm{p}$-value. Cohen's $\mathrm{D}$ was calculated using two times the $\mathrm{t}$ value divided by the square root of the degrees of freedom. 
1

\begin{tabular}{llllll}
\hline Parameter & $\begin{array}{l}\text { NIHSS } \\
\text { estimate (std.error) }\end{array}$ & $t(p)$ & Cohen's d & TOAST & location \\
\hline$K$ & $-0.066(0.063)$ & $-1.037(0.304)$ & -0.27 & $1.593(0.188)$ & $2.363(0.08)$ \\
$C$ & $0.408(0.977)$ & $0.418(0.678)$ & 0.109 & $1.937(0.116)$ & $0.756(0.523)$ \\
$t_{0}$ & $1.997(1.723)$ & $1.159(0.251)$ & 0.302 & $1.188(0.325)$ & $0.740(0.532)$ \\
error rate & $-0.003(0.007)$ & $-0.376(0.708)$ & -0.098 & $0.310(0.87)$ & $1.603(0.198)$ \\
\hline
\end{tabular}

2 Table 3. Summary statistics for the associations between each of the TVA parameters (dependent

3 variables) and each of the clinical measures. *Significant after Bonferroni correction. ${ }^{1}$ Main effect of

4 TVA parameters on Cogmed performance. $K=$ short-term memory capacity. $C=$ perceptual processing

5 speed. $t_{0}=$ perceptual threshold. NIHSS $=$ National Institute of Health Stroke Scale. TOAST $=$ Trial of

6 Org 10172 in Acute Stroke Treatment (TOAST) classification system. location = coarse four-class

7 classification of lesion location (left hemisphere, right hemisphere, bilateral and brain stem/cerebellum

8 lesions). Estimate $=$ unstandardized regression coefficients $b$. std.error $=$ standard error. $t=t$-value. $p=p$ -

9 value. Cohen's D was calculated using two times the $t$-value divided by the square root of the degrees of 10 freedom. 


\section{Table 4 (on next page)}

Summary statistics from the linear models testing for associations between Cogmed performance gain (dependent variable) and TVA performance at baseline, including age and sex as covariates.

*Significant after Bonferroni correction. ${ }^{1}$ Main effect of TVA parameters on Cogmed performance. $K=$ short-term memory capacity. $C=$ perceptual processing speed. $t_{0}=$ perceptual threshold. Estimate $=$ unstandardized regression coefficients $b$. std.error $=$ standard error. $\mathrm{t}=\mathrm{t}$-value. $\mathrm{p}=\mathrm{p}$-value. 
2

\begin{tabular}{lllllll}
\hline Parameter & $\begin{array}{l}\text { TVA } \\
\text { estimate } \\
\text { (std.error) }\end{array}$ & $t(p)$ & $\begin{array}{l}\text { age } \\
\text { estimate } \\
\text { (std.error) }\end{array}$ & $t(p)$ & $\begin{array}{l}\text { sex } \\
\text { estimate } \\
\text { (std.error) }\end{array}$ & $t(p)$ \\
\hline$K$ & $-0.33(0.304)$ & $-1.07(0.289)$ & $0.01(0.031)$ & $0.30(0.763)$ & $-0.42(0.507)$ & $-0.84(0.407)$ \\
$C$ & $-0.08(0.027)$ & $-2.79(0.007)^{*}$ & $-0.01(0.030)$ & $-0.46(0.645)$ & $-0.45(0.476)$ & $-0.95(0.344)$ \\
$t_{0}$ & $0.02(0.012)$ & $1.35(0.183)$ & $0.01(0.030)$ & $0.37(0.713)$ & $-0.33(0.512)$ & $-0.64(0.522)$ \\
error rate & $-3.21(2.717)$ & $-1.18(0.242)$ & $0.02(0.031)$ & $0.72(0.476)$ & $-0.36(0.512)$ & $-0.70(0.488)$ \\
\hline
\end{tabular}

3 Table 4. Summary statistics from the linear models testing for associations between Cogmed 4 performance gain (dependent variable) and TVA performance at baseline, including age and sex as

5 covariates. *Significant after Bonferroni correction. ${ }^{1}$ Main effect of TVA parameters on Cogmed

6 performance. $K=$ short-term memory capacity. $C=$ perceptual processing speed. $t_{0}=$ perceptual

7 threshold. Estimate $=$ unstandardized regression coefficients $b$. std.error $=$ standard error. $t=t$-value. $p=$ 8 -value. 


\section{Table 5 (on next page)}

Summary statistics from the linear mixed effects models testing for associations between TVA parameters (dependent variables) and group by session, including age and sex as covariates and participant as random factor.

*Significant after Bonferroni correction. $K=$ short-term memory capacity. $C$ = perceptual processing speed. $t_{0}=$ perceptual threshold. sess:group $=$ session by group interaction . Estimate $=$ unstandardized regression coefficients $b$. std.error $=$ standard error. $t=t$-value. $p$ $=p$-value. 
1

\begin{tabular}{llllll}
\hline Term & & $K$ & $C$ & $t_{0}$ & error rate \\
\hline \multirow{2}{*}{ session } & estimate (std.error) & $0.090(0.014)$ & $2.004(0.316)$ & $-2.502(0.427)$ & $0.006(0.002)$ \\
& $t(p)$ & $6.620(<.001)^{*}$ & $6.340(<.001)^{*}$ & $-5.860(<.001)^{*}$ & $3.180(0.002)^{*}$ \\
\multirow{2}{*}{ age } & estimate (std.error) & $-0.029(0.012)$ & $-0.666(0.169)$ & $0.167(0.248)$ & $0.001(0.001)$ \\
& $t(p)$ & $-2.410(0.019)$ & $-3.940(<.001)^{*}$ & $0.670(0.504)$ & $0.960(0.344)$ \\
\multirow{2}{*}{ sex } & estimate (std.error) & $-0.213(0.208)$ & $1.967(2.921)$ & $-1.147(4.286)$ & $0.007(0.021)$ \\
& $t(p)$ & $-1.020(0.311)$ & $0.670(0.504)$ & $-0.270(0.79)$ & $0.330(0.744)$ \\
group & estimate (std.error) & $0.003(0.194)$ & $1.113(2.994)$ & $2.005(4.301)$ & $-0.024(0.020)$ \\
& $t(p)$ & $0.020(0.987)$ & $0.370(0.712)$ & $0.470(0.643)$ & $-1.170(0.247)$ \\
sess:group & estimate (std.error) & $-0.018(0.019)$ & $0.787(0.446)$ & $-0.361(0.603)$ & $0.005(0.003)$ \\
& $t(p)$ & $-0.930(0.352)$ & $1.760(0.079)$ & $-0.600(0.550)$ & $1.730(0.085)$ \\
\hline
\end{tabular}

2 Table 5. Summary statistics from the linear mixed effects models testing for associations between TVA parameters (dependent variables) and group by session, including age and sex as covariates and participant as random factor. *Significant after Bonferroni correction. $K=$ short-term memory capacity. $C=$ perceptual processing speed. $t_{0}=$ perceptual threshold. sess:group $=$ session by group interaction. Estimate $=$ unstandardized regression coefficients $b$. std.error $=$ standard error. $t=t$-value. $p=$ $p$-value. 